\title{
Mid-Infrared Resonant Ablation of PMMA
}

\author{
Sanjeev NAITHANI ${ }^{1}$, Arnaud GRISARD ${ }^{2}$, David SCHAUBROECK ${ }^{1}$, Eric LALLIER $^{2}$, Geert Van STEENBERGE $^{1}$ \\ ${ }^{1}$ Centre for Microsystems Technology (CMST), imec and Ghent University, Technologiepark 914, \\ 9052 Gent, Belgium. \\ ${ }^{2}$ Thales Research \& Technology (TRT), Av. Augustin Fresnel 1, 91767 Palaiseau cedex, France. \\ E-mail: sanjeev.naithani@ugent.be
}

\begin{abstract}
Laser ablation proved to be a reliable micro-fabrication technique for patterning and structuring of both thin film and bulk polymer materials. In most of the industrial applications ultra-violet (UV) laser sources are employed, however they have limitations such as maintenance costs and practical issues. As an alternative and promising approach, mid-infrared resonant laser ablation (RIA) has been introduced, in which the laser wavelength is tuned to one of the molecular vibrational transitions of the polymer to be ablated. Consequently, the technique is selective in respect of processing a diversity of polymers which usually have different infrared absorption bands. In this paper, we present mid-infrared resonant ablation of PolyMethyl MethAcrylate (PMMA), employing nanosecond laser pulses tunable between 3 and 4 microns. This RIA nanosecond laser set-up is based on a commercial laser at $1064 \mathrm{~nm}$ pumping a singly resonant Optical Parametric Oscillator (OPO) built around a Periodically-Poled Lithium Niobate (PPLN) crystal with several Quasi-Phase Matching (QPM) periods. RIA has been successfully demonstrated for structuring bulk PMMA, and selective patterning of PMMA thin films on a glass substrate has been implemented.
\end{abstract}

DOI: $10.2961 / \mathrm{jlmn} .2014 .02 .0013$

Keywords: Laser machining, mid-infrared laser, PMMA, resonant ablation, tunable laser.

\section{Introduction}

A lot of scientific research work is devoted to polymer laser ablation $[1,2]$, driven by interest in the basic science as well as by numerous applications that have emerged for this high-resolution technique for material removal $[3,4]$. Due to the fact that this process is contactless, direct-write, can be employed at room temperature and atmospheric conditions, makes laser ablation a very attractive micromachining technology [5-7].

As most materials absorb very well in the UV and as shorter wavelengths correspond to a better optical resolution of the beam delivery system, UV excimer lasers are very suitable for patterning with micron and even submicron resolution $[8,9]$. This is in particular the case when the machined substrate is a polymer since this material features a low thermal conductivity, extremely high UV absorption, and the ability to decompose photo-chemically. During this photochemical ablation process, the incident photons have sufficiently high energy to directly break main chain bonds, restricting the temperature rise, and the extent of thermal damage to the substrate [10]. These UV lasers are suitable for structuring and patterning of thin films and particularly relevant for organic electronics. Ultraviolet lasers have proven their full potential for patterning single organic layers [11], but in a multilayer organic device the obtained layer selectivity is limited as all organic layers show high UV absorption.

That a purely thermal process can produce effective polymer ablation has been confirmed by a number of investigations using long wavelength $\mathrm{CO}_{2}$ lasers, in which the photons couple to vibrational modes of the molecule[12].
A promising approach named resonant infrared laser ablation (RIA) was introduced at the beginning of the $21 \mathrm{st}$ century[13]. This method uses a short pulse mid-infrared laser system, which can be wavelength tuned to one of the molecular vibrational transitions of the polymer to be ablated. As a result, the technique is selective in respect of ablating a diversity of polymers, which usually have different infrared absorption bands. So far most studies have been carried out using a free electron laser (FEL), providing picosecond pulses tunable between 2 and 10 microns $[13,14]$. However, in most applications, FELs are not practical since they are coupled to huge and expensive accelerators. Therefore, new technologies are being developed to replace FELs by bench-top solid-state photonic sources[15, 16]. Most of the resonant infra-red ablation (RIA) studies have been focused on the applications in thin film deposition [17, 18] and matrix-assisted laser desorption/ ionization mass spectroscopy $[19,20]$. However, the utilization of RIA in the patterning of thin films has not been reported so far. Moreover, RIA can be integrated with roll-to-roll (R2R) processes for patterning organics electronics.

In this paper, we present mid-infrared resonant ablation of PMMA employing nanosecond laser pulses tunable between 3 and 4 microns. This RIA nanosecond laser set-up is based on a commercial laser at $1064 \mathrm{~nm}$ pumping a singly resonant Optical Parametric Oscillator (OPO) built around a Periodically-Poled Lithium Niobate (PPLN) crystal with several Quasi-Phase Matching (QPM) periods, delivering more than $0.3 \mathrm{~W}$ of mid-IR power, corresponding to $15 \mu \mathrm{J}$ pulses. Moreover, mid-IR resonant ablation has been successfully implemented for patterning thin films of PMMA on a glass substrate, which provides a glimpse of 
using this technique for patterning applications, where selective removal is very crucial.

\section{Experimental work}

\subsection{Sample preparation}

Bulk sheets and thin films of PMMA were prepared for resonant and non-resonant mid-infrared ablation experiments. First, bulk sheets of PMMA (Good fellow, PMMA Acrylic sheet, $750 \mu \mathrm{m}$ ) were used for RIA. These PMMA sheets are pure and acrylic rubber modified. Next, in order to investigate selective laser patterning of thin films, samples with thicknesses of $650 \mathrm{~nm}$ and $1300 \mathrm{~nm}$ were prepared. For this application, a specific PMMA solution in anisole (950 PMMA A resist) was purchased from MICROCHEM technology (Germany). It is pure PMMA (molecular weight $\mathrm{M}_{\mathrm{w}}$ 950,000 g / mol.) dissolved in Anisole. The thin films were obtained by spin coating on a glass substrate $\left(2\right.$ inch $\times 2$ inch). Anisole $\left(\mathrm{Bp} 154^{\circ} \mathrm{C}\right)$ has been removed by evaporation at high temperature $\left(180^{\circ} \mathrm{C}\right.$, 90 s) on a hot plate immediately after spin coating.

\subsection{Infrared spectral analysis}

The infrared (IR) absorption spectra of the used PMMA materials were obtained by ATR-IR (Attenuated Total Reflection InfraRed) spectroscopy, using a Bio-Rad 575c FTIR spectrometer equipped with a golden gate module. The spectrum consists of 32 scans with a resolution of $4 \mathrm{~cm}^{-1}$.

\subsection{Mid-infrared ablation set-up}

The experimental mid-IR laser set-up, as schematically shown in Fig. 1, is based on a commercial laser at $1064 \mathrm{~nm}$ pumping with $\sim 15$ nanosecond pulses a singly resonant Optical Parametric Oscillator (OPO) built around a Periodically-Poled Lithium Niobate (PPLN) crystal with several Quasi-Phase Matching (QPM) periods.

In the OPO cavity, the signal can oscillate between $1500 \mathrm{~nm}$ to $1650 \mathrm{~nm}$, corresponding to idler wavelengths from $3660 \mathrm{~nm}$ to $3000 \mathrm{~nm}$. Coarse tuning can be obtained by translating the PPLN crystal, thus changing the QPM period. Wavelength fine tuning is achieved by heating the crystal, which allows us to achieve a continuous wavelength tuning between $3000 \mathrm{~nm}$ and $3600 \mathrm{~nm}$, by combining coarse and fine wavelength tuning.

Taking into account the various filters used after the OPO cavity to remove the remaining pump and signal photons, this OPO delivers more than $0.3 \mathrm{~W}$ of mid-IR power at $20 \mathrm{kHz}$ repetition rate, corresponding to $15 \mu \mathrm{J}$ pulses.

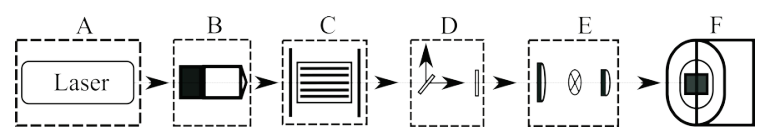

Fig. 1 Schematic diagram of the mid-IR ablation set-up consisting of six sub-divisions: (A) pump laser (B) attenuator / isolator (C) OPO / wavelength conversion unit (D) filtering section $(\mathrm{E})$ beam delivery unit $(\mathrm{F})$ motorized sample stage.

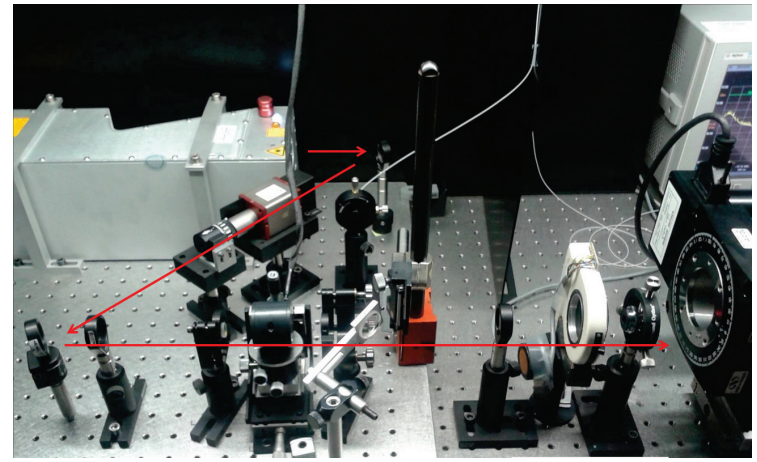

Fig. 2 Photograph of the bench-top mid-IR tunable high power ablation set-up (schematic is illustrated in Fig. 1).

The photograph of bench-top mid-IR laser ablation setup used in these experiments is shown in Fig. 2, in which the beam path is depicted by an arrow. The signal wavelength is projected to a free-space-fiber coupler, which is eventually connected to OSA (Optical Spectrum Analyzer). The signal wavelength is measured using an OSA and the corresponding idler wavelength is calculated. In the ablation set-up, the laser beam was focused on the target sample by a beam delivery system consisting of IR microscopic objective (Edmund Optics, $3.75 \mu \mathrm{m} \mathrm{CWL}, 6 \mathrm{~mm}$ FL ZnSe). The samples were mounted on a motorized rotational stage, allowing a controlled amount of laser shots per spot.

The ablation experiments for the bulk PMMA sheets were conducted for two non-resonant wavelengths (3.20 $\mu \mathrm{m}, 3.34 \mu \mathrm{m})$ and one resonant $(3.39 \mu \mathrm{m})$ wavelength. In case of PMMA thin film patterning on glass, the resonant $(3.39 \mu \mathrm{m})$ wavelength was selected. A pulse repetition rate of $20 \mathrm{kHz}, 165 \mathrm{~mW}$ output power, with different ablation speeds of 1 to $50 \mathrm{~mm} / \mathrm{s}$ were used during these experiments

\subsection{Depth profiles}

The ablated craters were inspected with an optical microscope. The crater depths were measured with a noncontact optical profiler (Wyko NT3300) and a mechanical profiler (Dektak 150).

\subsection{Scanning electron microscopy}

Scanning electron microscope (SEM) analysis was performed on a JEOL JSM-5600. The apparatus was used in the secondary electron mode. Prior to analysis, all samples were coated with a thin gold layer $(20 \mathrm{~nm})$ via plasma magnetron sputter coating.

\section{Results and discussions}

A detailed mid-IR absorption spectrum of PMMA obtained by ATR-IR spectroscopy is illustrated in Fig. 3. 


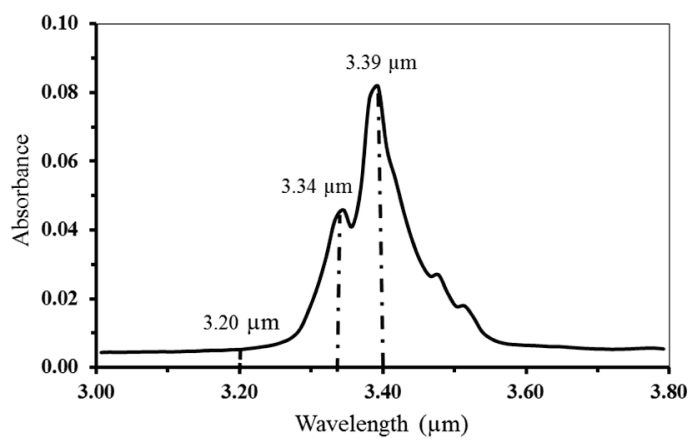

Fig. 3 Mid-infrared absorption spectrum of PMMA indicating a highest peak of absorption at $3.39 \mu \mathrm{m}$, a lower peak at $3.34 \mu \mathrm{m}$ and minimum absorption at $3.20 \mu \mathrm{m}$.

It is observed from this figure that there is a maximum peak of absorption at wavelength $3.39 \mu \mathrm{m}$ which corresponds to the $\mathrm{C}-\mathrm{H}$ asymmetric stretch in $\mathrm{CH}_{3}$. Next, a medium peak at $3.34 \mu \mathrm{m}\left(\mathrm{C}-\mathrm{H}\right.$ asymmetric stretch in $\left.\mathrm{CH}_{2}\right)$ is also noticed. Given the tunability of the laser, we are able to address both peaks, as well as a wavelength of 3.20 which corresponds to minimum absorption in this specific mid-IR range of PMMA.

Experiments on bulk PMMA sheets at the above mentioned resonant and non-resonant absorption wavelengths were performed with various amounts of laser shots. A comparative and qualitative study at three wavelengths has been carried out keeping all other laser parameters (fluence, pulse energy, focusing conditions) fixed.

The quality of the grooves obtained after mid-IR experiments at different wavelengths are shown in Fig. 4. After ablation at the lowest absorption wavelength $(3.20 \mu \mathrm{m})$ it is observed that the rim width is more pronounced and the ablated crater depth is shallow (See Fig. 4 (a)). As the wavelength is tuned to the medium absorption wavelength $(3.34 \mu \mathrm{m})$, the width and depth of the ablated crater increases (Fig. 4 (b)). Interestingly, few tiny particles in the ablated region are present which are attributed to trapped air bubbles during the re-solidification of material from the molten state. Finally, at the resonant wavelength $(3.39 \mu \mathrm{m})$, it can be clearly noticed (Fig. 4 (c)) that the ablated grooves width increases further. Moreover, the region is free of debris and / or re-deposited particles.

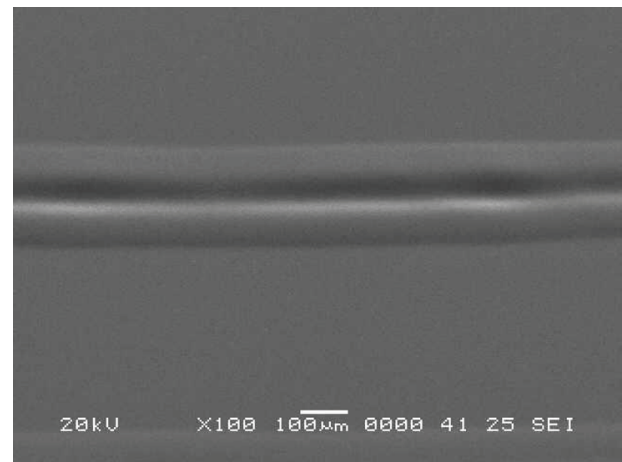

(a)

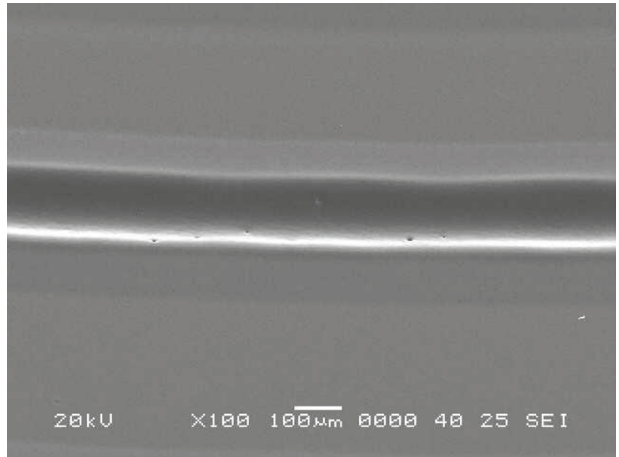

(b)

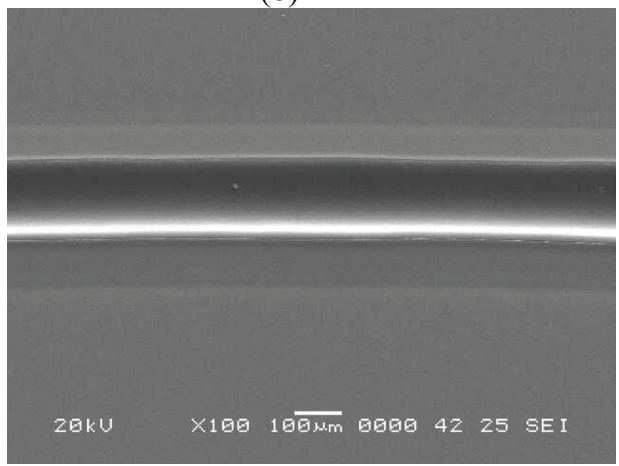

(c)

Fig. 4 SEM micrographs of PMMA mid-IR ablation at three wavelengths (a) $3.20 \mu \mathrm{m}$ (b) $3.34 \mu \mathrm{m}$ and (c) $3.39 \mu \mathrm{m}$.

By plotting the ablation depth versus translation speed, or amount of pulses, the ablation behavior of PMMA can be quantified. Results are summarized in Fig. 5 and Fig. 6 at these three different ablation wavelengths. Indeed, as expected, the influence of the wavelength on ablation is significant.

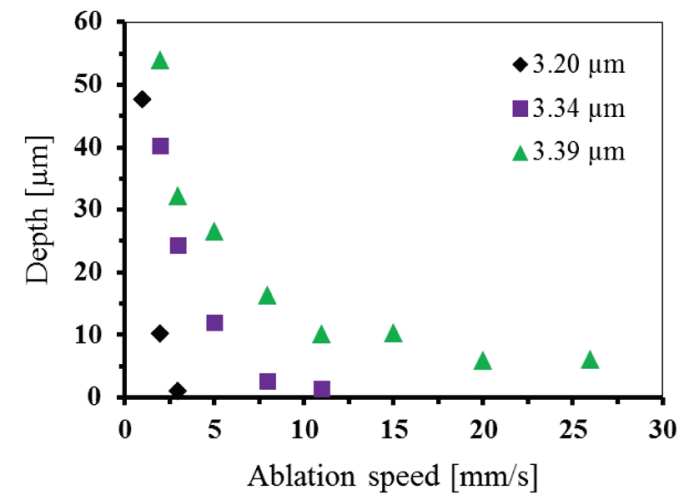

Fig. 5 Ablation depth plots of PMMA at three different wavelengths: $3.20 \mu \mathrm{m}, 3.34 \mu \mathrm{m}$ and $3.39 \mu \mathrm{m}$.

Now, if we compare the slopes in Fig. 6, the influence of the laser wavelength on the ablation rate (amount of material removed per laser pulse) is clearly noticeable. The ablation rate varies from $54 \mathrm{~nm}$ per laser pulse for nonresonant ablation to $77 \mathrm{~nm}$ per laser pulse for resonant ablation. Furthermore, the influence of laser wavelength on the incubation effect is pronounced. In general, incubation occurs when a weakly absorbing polymer material is converted to a material with a higher absorption cross-section after repetitive exposure through photo-chemical and/or photo-thermal reactions. 


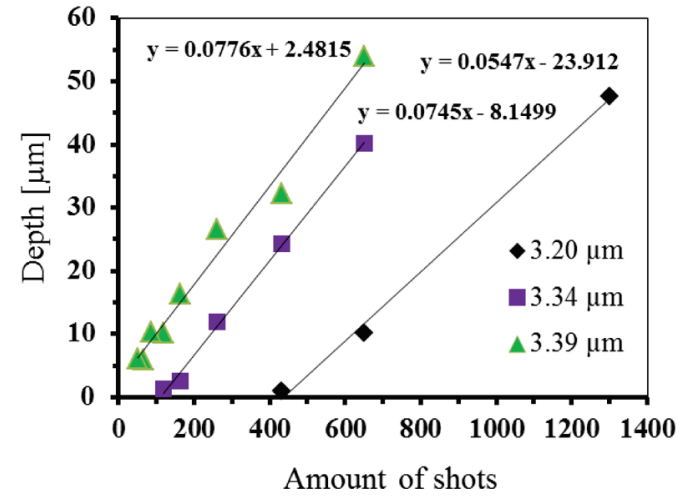

Fig. 6 Ablation rate plots for PMMA at three different mid-IR wavelengths of absorption: $3.20 \mu \mathrm{m}, 3.34 \mu \mathrm{m}$ and $3.39 \mu \mathrm{m}$.

For the resonant ablation the incubation effect is very limited, however in case of non-resonant wavelengths it is more pronounced. For instance from Figure 6, at $3.20 \mu \mathrm{m}$ wavelength with 400 shots per location the ablation depth is almost negligible compared to $24 \mu \mathrm{m}$ and $32 \mu \mathrm{m}$ ablation depths at $3.34 \mu \mathrm{m}$ and $3.39 \mu \mathrm{m}$ respectively.

This behavior of incubation and given tunability of RIA source can be exploited in case of selective laser patterning of a polymer on another polymer. For selective ablation, it is very important to remove the top layer without damaging the underneath layer (Fig. 7).

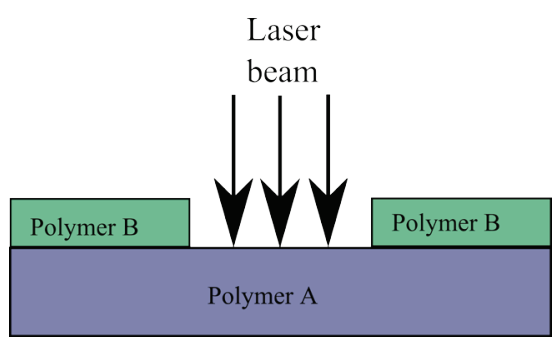

Fig. 7 Schematic diagram for selective ablation process of a polymer (Polymer B) on another polymer (Polymer A).

For the patterning applications, experiments were conducted at $20 \mathrm{kHz}, 165 \mathrm{~mW}$ and output wavelength was tuned to the resonant peak $(3.39 \mu \mathrm{m})$ of absorption. In this case, the laser fluence has been maximized by using a midIR microscopic objective (focal length $6 \mathrm{~mm}$ ). The laser spot size was not measured exactly, but estimated from crater depth profile as indicated in Figure 8. The calculated fluence for thin films patterning with this mid-IR ablation set-up and estimated spot size of $17.1 \mu \mathrm{m}$ (Full Width Half Maximum) is approximately $6.5 \mathrm{~J} / \mathrm{cm}^{2}$.

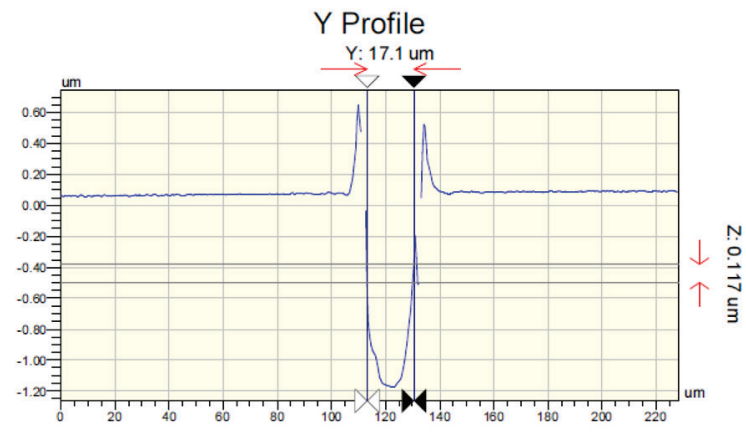

Fig. 8 Cross-section profile of an ablated structure indicating a spot size of $17.1 \mu \mathrm{m}$ at full-width-half-maximum

(FWHM).

The ablation results of PMMA thin films patterning on glass substrate are illustrated in Fig. 9. The optical images in Figure 9(a) and Figure 9(b), show clean and debris free ablated tracks with widths of $19 \mu \mathrm{m}$ and $22 \mu \mathrm{m}$ (including the rims). Next, the depth profiles shown in Figure 9(b) and Figure 9(d) indicate the removal of about $648 \mathrm{~nm}$ and 1298 nm respectively. These values are very close to the initial PMMA thin film thicknesses i.e. $650 \mathrm{~nm}$ and $1300 \mathrm{~nm}$.

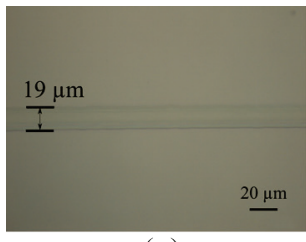

(a)

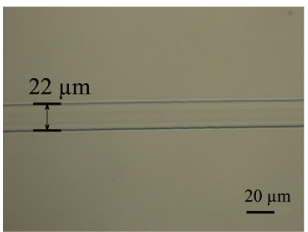

(c)

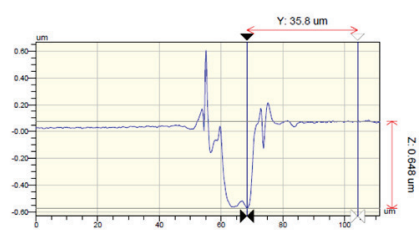

(b)

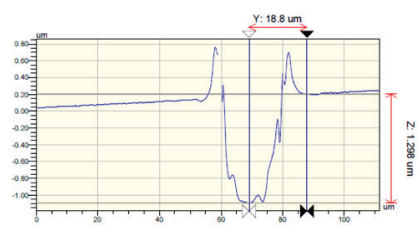

(d)
Fig. 9 Optical microscopic images: (a) and (c), of RIA patterned $650 \mathrm{~nm}$ and $1300 \mathrm{~nm}$ thin films PMMA on a glass. On the right: (b) and (d) are the corresponding depth profiles indicating a removal of $648 \mathrm{~nm}$ and $1298 \mathrm{~nm}$ of PMMA thin films.

It can be observed from Fig. 9 (b) and Fig. 9 (d) that there are ridges at the edges of the ablated area. There are two possible reasons for these ridges. First, the molecular weight of PMMA plays a significant role in the formation of ridges. It has been reported that there was bulge formation on rims for lower molecular weight PMMA, whereas no bulge formation for higher molecular weights [21]. In addition to this, the laser beam profile is also responsible for ridges, which is a Gaussian profile in this case. The energy at the sides of Gaussian beam is lower compared to the energy at beam axis and is also below the threshold value to ablate the material. This energy is absorbed near the surface, modifying surface chemistry, crystal structure, and /or multi-scale morphology without altering the bulk. These modifications at surface are followed by resolidification process, which leads to edges after laser patterning [22].

The SEM micrographs (Fig. 10) of the ablated regions with higher magnification (x1000) are clean and there is no visible re-deposition or debris on the ablated tracks.

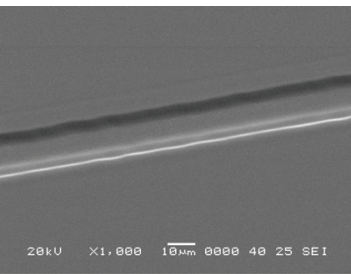

(a)

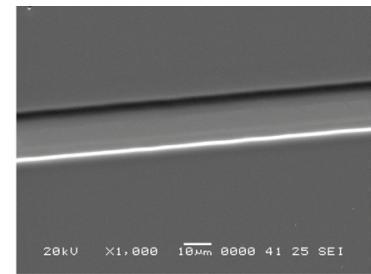

(b) 
Fig. 10 SEM micrographs of the thin films patterned PMMA (a) $650 \mathrm{~nm}$ (b) $1300 \mathrm{~nm}$. The micrographs are taken with 45 degree tilt and 1000 magnification.

It is inferred from these observations that the ablation is likely to be driven by photo-thermal mechanisms. The laser energy deposited into PMMA target is transformed into heat. Resonant infra-red $(3.39 \mu \mathrm{m})$ excitations of localized molecular-vibrations couple to the phonon within hundreds of fs to ps. This leads to heat accumulation in the material. The thermal energy can be diffused from the localized volume in its thermal confinement time. When the spot size of the laser beam is much larger than absorption length of the material to be ablated, one dimensional thermal diffusion can be assumed. In such a case, the thermal confinement time can be calculated by using the following equation [23]:

$$
\tau_{\text {th }}=c_{p} \rho / \alpha^{2} K
$$

Where $\tau_{\text {th }}$ is thermal confinement time, $c p$ is specific heat, $\rho$ is material density, $\alpha$ is the absorption coefficient and $K$ is thermal conductivity. Plugging some numerical values into this formula for PMMA, as $c_{p} \sim 1466 \mathrm{~J} / \mathrm{Kg}-\mathrm{K}$, $\rho \sim 1.18 \mathrm{~g} / \mathrm{cm}^{3}, \alpha \sim 1730 \mathrm{~cm}^{-1}$ and thermal conductivity of PMMA varies from 0.167 to $0.251 \mathrm{~W} / \mathrm{m}-\mathrm{K}$. This calculation leads to thermal confinement time of $\sim 0.29 \mathrm{~ms}$ (for $K$ $=0.200 \mathrm{~W} / \mathrm{m}-\mathrm{K})$, which is much higher than the laser pulse duration ( $\sim$ nanoseconds). Since the time required for the heat to diffuse out of the localized volume is much higher than the laser pulse length, thus this RIA ablation mechanism for PMMA assumed to be photo-thermal in nature.

\section{Conclusions}

A qualitative and quantitative analysis of the resonant mid-IR laser ablation (RIA) of bulk PMMA has been successfully demonstrated using a recently developed mid-IR tunable source. Higher ablation rates were observed at the resonant wavelength of operation and the ablated crater was free of debris. A proof of concept of thin film PMMA patterning on glass substrate has been provided.

\section{Acknowledgement}

This work was conducted in the framework of the project "IMPROV", funded within the European Commission FP7 program. This work is partly supported by the Research Foundation-Flanders through the project GA04711N.

\section{References}

R. Srinivasan, and B. Braren, "Ultraviolet -laser ablation of organic polymers," Chemical Reviews, 89(6), 1303-1316 (1989).

T. Lippert, "Interaction of photons with polymers: From surface modification to ablation," Plasma Processes and Polymers, 2(7), 525-546 (2005).

P. E. Dyer, R. J. Farley, R. Giedl et al., "Excimer laser ablation of polymers and glasses for grating fabrication," Applied Surface Science, 96-8, 537549 (1996).

[4] B. N. Chichkov, C. Momma, S. Nolte et al., "Femtosecond, picosecond and nanosecond laser ablation of solids," Applied Physics a-Materials Science \& Processing, 63(2), 109-115 (1996).

[5] J. Ihlemann, and B. WolffRottke, "Excimer laser micro machining of inorganic dielectrics," Applied Surface Science, 106, 282-286 (1996).

[6] J. Jandeleit, A. Horn, R. Weichenhain et al., "Fundamental investigations of micromachining by nano- and picosecond laser radiation," Applied Surface Science, 127, 885-891 (1998).

[7] P. Mannion, J. Magee, E. Coyne et al., "Ablation thresholds in ultrafast laser micromachining of common metals in air." Proc. of SPIE Vol.4876, 470-478 (2003).

[8] K. Naessens, H. Ottevaere, P. Van Daele et al., "Flexible fabrication of microlenses in polymer layers with excimer laser ablation," Applied Surface Science, 208, 159-164 (2003).

[9] M. Schaefer, J. Holtkamp, and A. Gillner, "Ablation of PEDOT/PSS with excimer lasers for micro structuring of organic electronic devices," Synthetic Metals, 161(11-12), 1051-1057 (2011).

[10] R. Srinivasan, and W. J. Leigh, "Ablative photodecomposition-action of far ultraviolet (193 nm) laser radiation on poly(ethyelene-terephthalate) films," Journal of the American Chemical Society, 104(24), 6784-6785 (1982).

[11] S. Naithani, D. Schaubroeck, Y. Vercammen et al., "Excimer laser patterning of PEDOT:PSS thin-films on flexible barrier foils: A surface analysis study," Applied Surface Science, 280, 504-511 (2013).

[12] J. H. Brannon, and J. R. Lankard, "Pulsed $\mathrm{CO}_{2}$ laser etching of polyimide," Applied Physics Letters, 48(18), 1226-1228 (1986).

[13] D. M. Bubb, J. S. Horwitz, J. H. Callahan et al., "Resonant infrared pulsed-laser deposition of polymer films using a free-electron laser," Journal of Vacuum Science \& Technology a-Vacuum Surfaces and Films, 19(5), 2698-2702 (2001).

[14] D. M. Bubb, J. S. Horwitz, R. A. McGill et al., "Resonant infrared pulsed-laser deposition of a sorbent chemoselective polymer," Applied Physics Letters, 79(17), 2847-2849 (2001).

[15] The European commission "IMPROV" project: http://www.fp7project-improv.eu (Website as viewed on 16/07/2013), (2013).

[16] M. Duering, R. Haglund, B. Luther-Davies et al., "Resonant Infra-red Pulsed Laser Ablation of Polymers with single picosecond pulses generated by an optical parametric amplifier" IEEE Conference on Lasers and Electro-Optics (CLEO), New York (2010).

[17] S. Chaudhuri, M. R. Nevala, T. Hakkarainen et al., "Infrared Pulsed Laser Deposition of Niobium Nitride Thin Films," Ieee Transactions on Applied Superconductivity, 21(3), 143-146 (2011).

[18] D. M. Bubb, B. Toftmann, R. F. Haglund et al., "Resonant infrared pulsed laser deposition of thin 
biodegradable polymer films," Applied Physics aMaterials Science \& Processing, 74(1), 123-125 (2002).

[19] W. P. Hess, H. K. Park, O. Yavas et al., "IRMALDI of low molecular weight compounds using a free electron laser," Applied Surface Science, 127, 235-241 (1998).

[20] M. Sadeghi, Z. Olumee, X. D. Tang et al., "Compact tunable Cr:LiSAF laser for infrared matrix-assisted laser desorption/ionization," Rapid Communications in Mass Spectrometry, 11(4), 393-397 (1997).

[21] Y. C. L. Nimai, C. Nayak, C. Y. Yue and Ayan T. Sinha, " $\mathrm{CO}_{2}$-laser micromachining of PMMA: the effect of polymer molecular weight" Journal of micromechanics nad microengineering 18 ( 095020), 7 (2008).

[22] M. S. Brown and C. B. Arnold, "Fundamentals of Laser-Material Interaction and Application to Multiscale Surface Modification" Springer Series in Materials Science, Springer-Verlag Berlin Heidelberg (2010).

[23] S. L. Johnson, D. M. Bubb, and R. F. Haglund, "Phase explosion and recoil-induced ejection in resonant-infrared laser ablation of polystyrene," Applied Physics A -Materials Science \& Processing, 96(3), 627-635 (2009).

(Received: August 19, 2013, Accepted: April 28, 2014) 